Introduction: Cancer cachexia is a common associate of cancer and has a negative impact on both patients' quality of life and overall survival. Nonetheless its management remains suboptimal in clinical practice. Provision of medical recommendations in websites is of extreme importance for medical decision making and translating evidence into clinical practice.

Aim of the study: To scrutinize the magnitude, consistency and changes over time of cancer-cachexia recommendations for physicians on the Web among oncology related societies. Intercontinental, continental, national and socioeconomic variations were further analyzed.

Material and methods: Web identification of oncology related societies and prospective analyses of relative Web guideline recommendations for physicians on cancer-cachexia at different time-points.

Results: In June 2011, we scrutinized 144,000 Web pages. We identified 275 societies, of which 270 were eligible for analyses: 67 were international (African, American, Asian, European, Oceania and Intercontinental), 109 belonged to the top 10 countries with the highest development index and 94 pertained to 10 countries with a long lasting tradition in medical oncology.

Conclusions: The magnitude of cancer cachexia recommendations for physicians on the Web at a global level was scant both for coverage and consistency, and at any time-point considered: $3.7 \%(10 / 270)$ in 2011 and $8.1 \%$ $(22 / 270)$ in 2018 . The proportion of societies giving evidence-based and updated recommendations for cancer cachexia for physicians was only $1.1 \%$ $(3 / 270)$ in 2011 and $2.96 \%(8 / 270)$ in 2018. Continent, national highest developmental index, oncology tradition and economic-geographic areas were not found to influence Web guideline provision.

Key words: cancer cachexia, globa awareness, guideline implementation, Web, medical societies, medical providers, oncology.

Contemp Oncol (Pozn) 2019; 23 (2): 100-109 DOI: https://doi.org/10.5114/wo.2019.85882

\section{Global coverage and consistency of guideline recommendations for cancer cachexia on the Web in 2011 and 2018}

\author{
Davide Mauri ${ }^{1}$, Georgia Zafeiri ${ }^{1}$, Melina Yerolatsite ${ }^{1}$, Lampriani Tsali ${ }^{2}$, \\ Georgios Zarkavelis ${ }^{1}$, Anna Tsiara ${ }^{3}$, Nikolaos P. Polyzos ${ }^{4}$, Antonis Valachis ${ }^{5}$, \\ Konastantina Kalopita ${ }^{6}$, Eleftherios Kampletsas ${ }^{1}$, Alexandra Papadaki ${ }^{1,7}$, \\ Evangelia Peponi ${ }^{8}$, Fani Kapoulitsa ${ }^{1}$, Panagiotis Filis ${ }^{1}$, \\ Georgios Pentheroudakis ${ }^{1,7}$
}

\author{
'Department of Medical Oncology, University Hospital of Ioannina, Greece \\ 2Department of Internal Medicine, General Hospital of Arta, Arta, Greece \\ 3Department of Medical Oncology, Alexandra General Hospital, Athens, Greece \\ ${ }^{4}$ Hospital Universitari Dexeus, Barcelona, Spain \\ ${ }^{5}$ Centre for Clinical Research Sörmland, Uppsala University, Uppsala, Sweden \\ ${ }^{6}$ Department of Anaesthesiology and Pain Medicine, Alexandra General Hospital, \\ Athens, Greece \\ EMEKEN, Ioannina, Greece \\ ${ }^{8}$ Department of Radiotherapy, University Hospital of Ioannina, Greece
}

\section{Introduction}

Cachexia and malnourishment are common events during treatment of cancer patients. Overall one third of patients with malignancy suffer from cancer cachexia, this proportion being notably higher among patients affected by solid cancers, with a prevalence of $60-80 \%$ in advanced cancers [1-4].

Cancer cachexia severely jeopardizes patients' quality of life and performance, and substantially contributes to morbidity and mortality of treatable cancers. It is an independent predictor of shorter survival and it increases the risk of treatment failure, and toxicity. It was estimated that cancer cachexia may directly contribute to $30 \%$ of cancer deaths [3-6].

However, in spite of its frequency and its severe negative impact on patients' performance and clinical outcomes, cancer cachexia screening, assessment and treatment are frequently less than satisfactory in daily oncology practice $[2,7,8]$. Medical oncologists and other healthcare oncology professionals treating patients with cancer seem indeed to neglect patients' nutritional issues, any setting considered [8-10]. This was one of the main factors that led the European Cancer Patient Coalition to publish a Cancer Patient's Nutritional Bill of Rights, which was presented in the European Parliament in Brussels in November 2017 [11].

A crucial question to answer is why this low level of priority in cancer cachexia management exists in daily clinical practice.

Considering that clinical practice guidelines are important for translating evidence into medical decision making and clinical practice applications, reducing undesirable practices and encouraging services of proven efficacy [12], we hypothesized that one of the possible causes of current medical mismanagement of cachectic patients might stem from a low level of cancer-cachexia guideline recommendations among oncology educational and policymaker societies/institutions at a global level [13]. Indeed, medical recommendations' delivery in websites has been documented to be of extreme importance, since it improves patient safety, reduces complications and shortens length of stay among Medicare beneficiaries [14]. 
In 2011 we launched a Web-based study with the aim of examining the global intercontinental coverage and consistency of Web guidelines for cancer cachexia produced by oncology related professional societies and their changes over time [13]. In our first analyses (June 2011) data from 275 oncology societies/health providers were scrutinized. The magnitude of "updated" and "evidence-based" cancer cachexia guideline recommendations on the Web among oncology societies was found to be extremely low at a global level irrespectively of continent, nation, developmental index, and type of oncology providers scrutinized [13]. We therefore concluded that the low level of global Web recommendations could be one cause of the observed low rank of priority given to the management of cancer cachexia in daily oncology practice.

Overall the number of guideline recommendations on the net from medical societies (any setting considered), and the use of internet and medical websites from physicians had an exponential growth in the last decade, giving a comprehensive picture of flourishing medical activities and scientific progress. But what happened with cancer cachexia?

We here report the June 2018 update of our study. Both magnitude of global Web recommendations for cancer cachexia for physicians among oncology related societies and their trend to change overtime (2011 vs. 2018) are analyzed.

\section{Material and methods}

\section{Identification of pertinent societies and caregivers}

In 2011, 144,000 Web pages were scrutinized during internet searches in order to identify oncology societies/ organizations that might have provided Web guidelines regarding cancer cachexia [13] (Appendix 1). We considered societies and organizations that were intercontinental (with a global outlook), continental (including two or more countries in the same continent: African, Asian, European, Oceanian, North American, South American), or national belonging to one of the top 10 countries with the highest development index (Norway, Australia, New Zealand, USA, Ireland, Liechtenstein, Netherlands, Canada, Sweden, Germany) [15]. Countries with a long lasting tradition in medical oncology but not included in the top 10 highly developed countries (Austria, Belgium, China, Denmark, France, Japan, Italy, UK, Spain, Switzerland) were further included in the internet searches (Table 1). Due to notable economic and development differences between South and North American countries, the continental entities were separately analyzed for North and South America.

Since guideline release may be influenced by each nation's economics and traditions, the national guidelines retrieved were further shared in groups by economicgeographic area: Australia-New Zealand vs. Benelux (Belgium and Netherland) vs. German speaking countries (Austria, Germany, Liechtenstein, Switzerland) vs. North American (US and Canada) vs. Scandinavian (Denmark, Norway and Sweden) vs. South European (France, Italy and Spain) vs. United Kingdom vs. East Asian (Japan and China).

We further constructed a database of 275 oncology related educational and policymaker societies, caregivers, and organizations that might provide guidelines for cachexia in cancer patients [13]. Of these, 4 ceased and one was the Spanish duplicate of $\mathrm{NCl}$, leaving 270 oncology societies/policymakers eligible for comparative analyses between 2011 and 2018 (Appendix 2). Relative websites were thereafter scrutinized for cancer cachexia/malnutrition guideline recommendations both in June 2011 and in June 2018 (Fig. 1).

Analyses were performed in ITT fashion; thereafter all the 270 eligible societies were scrutinized independently of the Web page accessibility (having no functional Web page, having Web page under construction, having no webpage, having no e-link active) at the time of analyses (June 2011 and June 2018).

\section{Primary outcome}

To scrutinize the global magnitude of "updated" and "evidence-based" guideline recommendations for cancer cachexia for physicians on the Web and its changes over time. We considered as "updated" all the Web guidelines that have been produced or revised or lastly adjourned within the last five years. Evidence-based guidelines were considered to be all those including randomized controlled trials and/or meta-analyses in references to support sentences.

\section{Secondary outcome}

To depict the global attitude towards cancer cachexia Web recommendations/guidelines (any level of evidence, any target).

Since all medical societies may not have the possibility to produce "internal" guidelines ("own" guidelines), we considered of value both "own" produced guidelines and/or those provided as a "link" to a specific website of another society with recommendations for cancer cachexia.

\section{Results}

\section{Eligible societies and organizations}

Overall 275 oncology-related educational and policymaker societies were registered in 2011 [13], and 270 of these were eligible for comparative analyses and were scrutinized for cancer cachexia guideline recommendations both in June 2011 and in June 2018 (Appendix 2).

Among these, 67 were international (23 intercontinental and 44 continental: African, American, Asian, European, Oceania), 109 belonged to the top 10 countries with the highest development index available in 2011 [15] and 94 pertained to countries with a long lasting tradition in medical oncology but not included in the top 10 highly developed countries. Searches for North America did not lead to comprehensive (US + Canada) North American societies/organizations and only societies for each separate country were retrieved and scrutinized. The retrieved 
Table 1. Demographics of the scrutinized societies and caregiver organizations

\begin{tabular}{|c|c|c|c|c|c|c|}
\hline Demographics & $\begin{array}{c}\text { All } \\
n=275\end{array}$ & $\begin{array}{l}\text { Eligible } \\
n=270\end{array}$ & $\begin{array}{c}2011 \text { any recomm. } \\
\text { cachexia } \\
n=10\end{array}$ & $\begin{array}{c}2018 \text { any recomm. } \\
\text { cachexia } \\
n=22\end{array}$ & $\begin{array}{c}2011 \text { EBU guidelines } \\
\text { cachexia } \\
n=3\end{array}$ & $\begin{array}{c}2018 \text { EBU guidelines } \\
\text { cachexia } \\
n=8\end{array}$ \\
\hline \multicolumn{7}{|l|}{ Continent } \\
\hline Intercontinental & 26 & 23 & 0 & 1 & 0 & 0 \\
\hline North America & - & - & - & - & - & - \\
\hline South America & 5 & 4 & 0 & 0 & 0 & 0 \\
\hline Europe & 24 & 24 & 2 & 5 & 2 & 1 \\
\hline Africa & 10 & 10 & 0 & 0 & 0 & 0 \\
\hline Asia & 4 & 4 & 0 & 0 & 0 & 0 \\
\hline Oceania & 2 & 2 & 0 & 0 & 0 & 0 \\
\hline \multicolumn{7}{|c|}{ Top 10 developed countries* } \\
\hline Norway & 3 & 3 & 0 & 0 & 0 & 0 \\
\hline Australia & 12 & 12 & 0 & 0 & 0 & 0 \\
\hline New Zealand & 6 & 6 & 0 & 0 & 0 & 0 \\
\hline USA & 46 & 45 & 3 & 6 & 1 & 3 \\
\hline Ireland & 9 & 9 & 0 & 0 & 0 & 0 \\
\hline Liechtenstein & 0 & 0 & 0 & 0 & 0 & 0 \\
\hline Netherlands & 8 & 8 & 0 & 0 & 0 & 0 \\
\hline Canada & 16 & 16 & 1 & 1 & 0 & 1 \\
\hline Sweden & 3 & 3 & 1 & 0 & 0 & 0 \\
\hline Germany & 7 & 7 & 0 & 0 & 0 & 0 \\
\hline \multicolumn{7}{|l|}{ Other countries } \\
\hline Japan & 11 & 11 & 0 & 0 & 0 & 0 \\
\hline United Kingdom & 13 & 13 & 2 & 2 & 0 & 0 \\
\hline Italy & 9 & 9 & 1 & 2 & 0 & 1 \\
\hline Switzerland & 12 & 12 & 0 & 0 & 0 & 0 \\
\hline Spain & 10 & 10 & 0 & 2 & 0 & 1 \\
\hline Belgium & 6 & 6 & 0 & 1 & 0 & 0 \\
\hline Denmark & 4 & 4 & 0 & 0 & 0 & 0 \\
\hline France & 9 & 9 & 0 & 0 & 0 & 0 \\
\hline China & 12 & 12 & 0 & 2 & 0 & 1 \\
\hline Austria & 8 & 8 & 0 & 0 & 0 & 0 \\
\hline \multicolumn{7}{|c|}{ Nations by economic-geographic area } \\
\hline Australia - New Zealand & 18 & 18 & 0 & 0 & 0 & 0 \\
\hline Benelux & 14 & 14 & 0 & 1 & 0 & 0 \\
\hline Germanophone & 27 & 27 & 0 & 0 & 0 & 0 \\
\hline North America & 62 & 61 & 4 & 7 & 1 & 4 \\
\hline Scandinavian & 10 & 10 & 1 & 0 & 0 & 0 \\
\hline South European & 28 & 28 & 1 & 4 & 0 & 2 \\
\hline UK-Ireland & 22 & 22 & 2 & 2 & 0 & 0 \\
\hline East Asian & 23 & 23 & 0 & 2 & 0 & 1 \\
\hline \multicolumn{7}{|l|}{ Society type } \\
\hline Cancer research & 52 & 52 & 1 & 1 & 0 & 0 \\
\hline Radiation oncology & 34 & 34 & 0 & 0 & 0 & 0 \\
\hline Medical oncology & 25 & 25 & 0 & 5 & 0 & 4 \\
\hline Surgical oncology & 15 & 15 & 0 & 0 & 0 & 0 \\
\hline Supportive care & 10 & 10 & 2 & 2 & 2 & 0 \\
\hline Comp. cancer manag. & 71 & 71 & 3 & 7 & 1 & 3 \\
\hline Other & 63 & 63 & 4 & 7 & 0 & 1 \\
\hline
\end{tabular}

EBU - evidence-based and updated, * Countries were selected from the top 10 countries according to the human development index available in 2011, Comp. cancer manag. - comprehensive cancer management; economo-geographic area: Australia-New Zealand vs. Benelux (Belgium and Netherland) vs. German speaking countries (Austria, Germany, Liechtenstein, Switzerland) vs. North American (US and Canada) vs. Scandinavian (Denmark, Norway and Sweden) vs. South European (France, Italy and Spain) vs. United Kingdom vs. East Asian (Japan and China) 


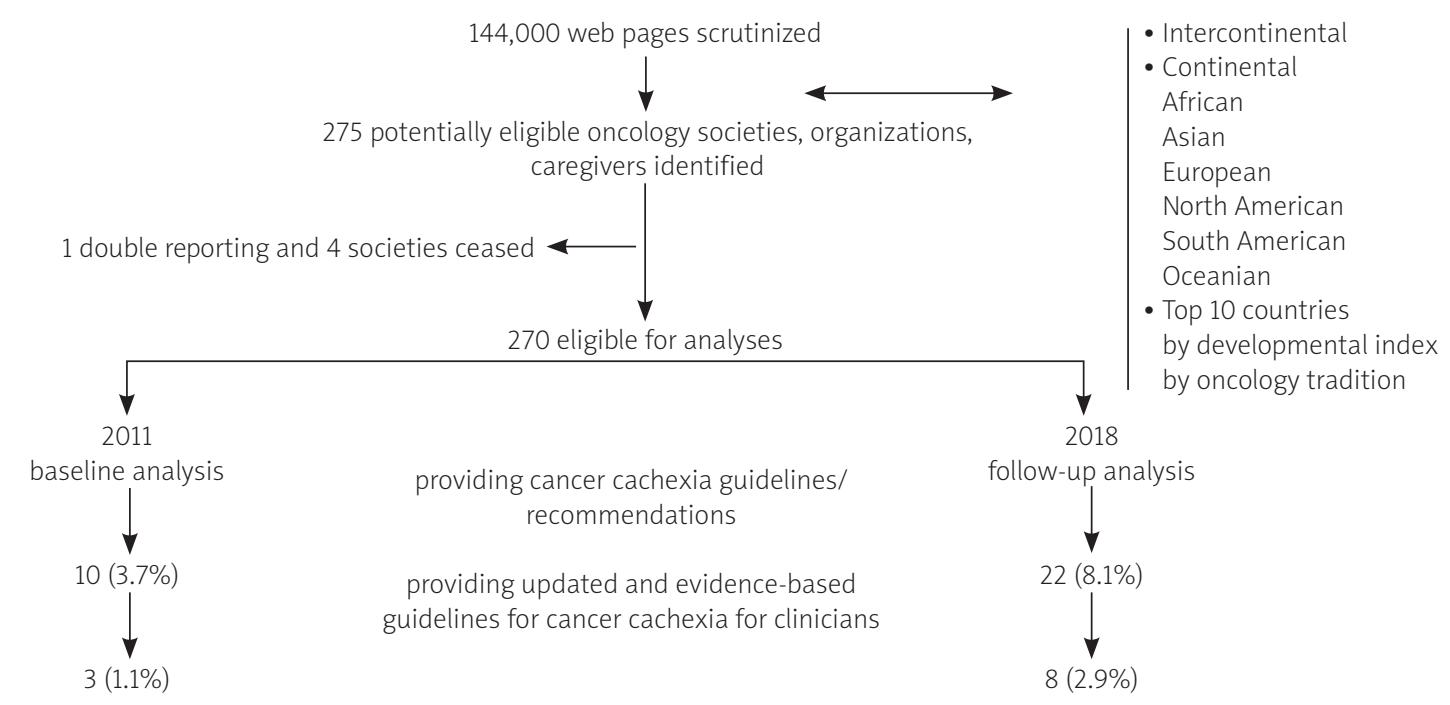

Fig. 1. Flow chart diagram of our research

and analyzed oncology societies and organizations covered a large array of oncology settings (educational/clinical/research/policymaker): most societies were devoted to comprehensive cancer management $(n=71)$, cancer research $(n=52)$, radiation oncology $(n=34)$ and medical oncology $(n=25)$, while only a minority pertained to surgical oncology $(n=15)$ and supportive oncology $(n=10)$ (Table 1, Fig. 1).

\section{Coverage of Web global recommendations on cancer cachexia}

Despite the extensive search and the notable number of societies organization eligible for analyses $(n=270)$, we found only 10 societies/organizations giving cachexia recommendations in 2011 [16-25] and only 22 societies in 2018 [26-47]. Thus, since the paucity of events may have led to unreliability of statistical comparison within categories, descriptive statistics were adopted when $\chi^{2}$ and Yates' $\chi^{2}$ tests were not applicable.

\section{Overall Web recommendations provision}

We found a statistically significant increase $\left(\chi^{2} p=\right.$ 0.0287 ) in the proportion of oncology societies/organizations providing recommendations for cachexia between 2011 and 2018 (3.7\% vs. 8.1\%) [16-47].

\section{Primary outcome}

When only updated and evidence-based guidelines were analyzed (guidelines updated within five years and with references including randomized controlled trials and/or meta-analyses to support sentences), we found that the proportion of oncology societies implementing cancer cachexia guidelines for physicians was almost nil both in 2011 and 2018 (1.1\% vs. 2.96\%) (Table 1). Indeed, only three $[17,20,21]$ and eight $[28,32,33,35,38,39,45$, 47] societies provided level one evidence-based and updated cancer-cachexia guideline for physicians in 2011 and 2018 respectively $\left(\chi^{2} p=0.1277\right.$, Yates' $\left.\chi^{2} p=0.2229\right)$. Nonetheless, consistency of these recommendations no- tably varies among scrutinized providers: from some paragraphs in the framework of supportive care guidelines [33], to extended and detailed guidelines [35, 38, 39, 45].

Evidence-based and updated guidelines for both cancer cachexia assessment and management were provided by two societies in 2011 [20, 21] and by seven societies in 2018 $[28,32,35,38,39,45,47]$ while one organization produced recommendations mainly for cancer cachexia management [17, 33].

Almost all societies produced their own guidelines/ recommendations both in 2011 and in 2018 [17, 20, 28, 32, $33,35,45,47$ ], while one organization in 2011 [21] and two societies in 2018 were presenting cancer cachexia guideline/recommendations by link to guidelines produced by other societies [38] or a consensus panel [39].

\section{Web guideline provision by geographic areas \\ International societies}

No recommendations were found among the scrutinized Asian, African Oceanian, and South American societies. Most cancer cachexia Web recommendations/guidelines were provided by European oncology societies [20, 21, 27-31]. However, even the overall European guideline provision was notably low (Table 1). Thus, the comprehensive international guideline release was inconsistent and not influenced by the continent analyzed (intercontinental vs. African vs. Asian vs. European vs. Oceanian vs. South American) both for primary and secondary outcome, both in 2011 and 2018 (Table 1).

\section{National societies}

The level of cancer cachexia recommendations and guidelines was almost zero across the different national oncology societies scrutinized both in 2011 and 2018. Paucity of evidence-based guidelines and/or overall cachexia recommendations was independent of the high developmental index of the nation and the oncology tradition. Most of the national guidelines were produced by Chinese $[46,47]$, Italian [25, 44, 45], Spanish [39, 40], and US soci- 
eties [16-18, 32-37]. Societies from most nations analyzed do not provide any evidence-based and updated guideline either in 2011 or 2018 (Table 1).

When national guidelines were analyzed by economic-geographic area, we found that guideline production was higher among countries from North American and Southern European economic-geographic areas, but the observed differences were not statistically significant (Table 1).

\section{Web guidelines provision by society type}

Analyses for society type did not translate into any recommendation difference at any time point (2011 vs. 2018) either for overall recommendations for cancer cachexia (2011 Yates' $\chi^{2} p=0.54 ; 2018$ Yates' $\chi^{2} p=0.29$ ) or for evidence-based and updated cachexia guidelines for physicians (2018 Yates' $\chi^{2} p=0.065,2011$ almost null recommendation and statistics not applicable) (Table 1).

However, a major change should be underscored both for major societies for comprehensive cancer management and for major medical oncology societies. In 2011 no guidelines at any level were retrieved from medical oncology, radiation oncology or surgical oncology societies/organizations. In 2015 ASCO (the American Society of Clinical Oncology) included a chapter for the management and assessment of cancer cachexia in its educational book [32]. ESMO (the European Society of Medical Oncology), at the time of data extraction in 2018, was producing its official guidelines, and in its website has a ppt module for cachexia e-learning (last adjourned in 2017) [27]. All major societies involved in comprehensive cancer management, $\mathrm{NCl}, \mathrm{NCCN}, \mathrm{ESO}$ provided their own evidence-based and updated guideline in 2018 [28, 33, 35]. Surprisingly, still in 2018, surgical oncology and radiation oncology societies do not provide any guideline for cancer cachexia (Table 1).

\section{Discussion}

An impressive number of cancer societies, cancer organizations and oncology policymakers have been developed over time, offering a general picture of flourishing professional and scientific activity.

We scrutinized 270 cancer societies/organizations that operate at the international or national level both in 2011 and 2018. Among these, we found that the provision of Web guideline recommendations for cancer cachexia for physicians was extremely poor at any time point analyzed. Paucity of Web guideline recommendation was found to be a global phenomenon and it was independent of the continent analyzed (Africa, America, Asia, Europe, Oceania), nation analyzed, high developmental index of the nation analyzed, oncological tradition of the country, and national grouping by economic-geographic area (Australia-New Zealand vs. Benelux vs. German speaking countries vs. North American vs. Scandinavian vs. Southern European vs. United Kingdom vs. East Asian).

Overall, ten (3.7\%) of the scrutinized societies were providing some form of recommendations in 2011 and twenty-two (8.1\%) in 2018. Nonetheless, consistency of provided guidelines was disappointingly scant. Only three societies
(1.1\%) in 2011 and 8 societies (2.96\%) in 2018 were providing updated and evidence-based guideline for physicians for the assessment and management of cancer cachexia. Guideline consistency by both evidence provision and continual updating is not a redundant issue. Evidence-based recommendations reduce undesirable practices and encourage services of proven efficacy $[12,14]$. In turn, timelines of guideline updates is crucial, since the implementation of outdated guidelines in clinical practice may lead to a lack of updated clinical decisions and practices.

Thus we found that the global provision of Web guidelines for cancer cachexia was extremely inadequate both for coverage and consistency.

A crucial question to answer is why this low level of priority in provision of guideline recommendation for cancer cachexia exists.

Cancer cachexia is a common event during treatment of cancer patients. It may reach prevalence of $60-80 \%$ in advanced cancers [1-4], with severe implications for quality of life, occupational possibilities and treatment outcomes, directly contributing to $30 \%$ of cancer deaths [3-6, 48].

Nonetheless, medical oncologists and other healthcare professionals treating patients with cancer seem to neglect patients' nutritional issues [8-10, 48]. This should not surprise and underscores an astonishing European report for cancer pain management where half of the patients believed that their quality of life was not considered a priority in their overall care by their health care professionals [49].

Does the reported paucity of guidelines explain the level of awareness of cancer cachexia in daily clinical practice? Probably yes, it may constitute a cause, since medical recommendations' delivery in websites has been documented to be of extreme importance in enhancing the implementation of practices of proved evidence in daily clinical activities [14].

Is there a light at the end of the tunnel? In 2011 no guidelines at any level were retrieved from strict medical oncology, radiation oncology or surgical oncology societies/organizations. In 2018 still no investigated surgical oncology societies (including ESSO, SSO) and radiation oncology societies (including ESTRO, ASTRO) provided any guideline for cancer cachexia. However, in the face of the general discouraging global scarcity in guideline provision, we found that both major societies for comprehensive cancer management and major medical oncology societies (ASCO, ESMO, $\mathrm{NCCN}, \mathrm{NCl}, \mathrm{ESO}$ ) were providing or were going to provide evidence-based recommendations on their websites. This last finding may be of extraordinary importance and may constitute a cornerstone for better management of cachexia and nutritional derangements in the near future. Indeed, all of these organizations have very extensive membership, organize large meetings and have a substantial influence upon their members, subscribers, and visitors to the websites. Nonetheless, the overall global provision of guidelines continues to be very scant.

We have to discuss some limitations of our study. Firstly, since there are no established validated searches for unearthing professional societies and organizations, some of them may have been missed by our searches. How- 
ever, given the multiple layers of our search, and the large number or oncology societies retrieved, it is unlikely that prominent oncologic entities were missed and that missed societies may change the global patterns of Web guideline provision for cancer cachexia [13, 50-52]. Secondly, our searches were oncology oriented, and thus some nutritional (non-oncology) societies with nutritional guidelines such as ESPEN (European Society for Clinical Nutrition and Metabolism) have been lost by our searches. However, it is unlikely that medical oncologists, radiation oncologists, and surgical oncologists (the physician gatekeeper for the oncology patients) will routinely visit nutritional societies' websites in their daily practice to take care of their patients. Thus, for nutritional societies, we consider as a "guideline-presence event" only a Web link to these guidelines from the scrutinized 270 societies. Finally, the human development index (HDI) changes over time. Thus, in June 2018 (at the time of data extraction) [53], countries' position variations as compared to the top 10 available in June 2011 were reviewed [15, 53]. Among the 188 nations analyzed by the $\mathrm{HDI}$, seven countries included in the top 10 for HDI at time of our analyses in 2011 (Norway, Australia, USA, Ireland, Netherland, Canada, Germany) [15] continued to be in the top 10 at the time of our data extraction in June 2018 [53]. The remaining three countries continue to rank at the top of the list, all included in the top 15 positions (New Zealand 13/188, Sweden 14/188, and Liechtenstein 15/188) [53]. Therefore, no significant biases may be attributed to the country with the highest developmental national index migration at the two time-points of analyses.

\section{Conclusions}

Cancer cachexia global awareness among oncology societies seems to be extremely low since related level guidelines implementation was found to be inconsistent both for coverage and consistency at any level and timepoint analyzed (nation vs. continent vs. international vs. economic-geographic area vs. oncology society type vs. 2011 and 2018). Some lights of hope seem to appear at the end of the tunnel since some major oncology societies have provided or are still developing some guidelines or educational material for the management of cancer cachexia on their websites. A lot of work has to be done in guidelines provision for cancer cachexia, in order to improve clinical management of cachectic patients in daily oncology practice.

The authors declare no conflict of interest.

\section{References}

1. Fabbro E, Inui A, Strasser F. Cancer cachexia. Pocket book for cancer supportive care. Springer Healthcare 2012.

2. von Haehling S, Anker SD. Cachexia as major underestimated and unmet medical need: facts and numbers. I Cachex Sarcopenia Muscle 2010: 1: 1-5.
3. Muscaritoli M, Bossola M, Aversa Z, Bellantone R, Rossi Fanelli F. Prevention and treatment of cancer cachexia: new insights into and old problem. Eur J Cancer 2006; 42: 31-41.

4. Cancer Cachexia Hub. Epidemiology. http://www.cancercachexia. com/epidemiology-hcp (lastly accessed 10 $0^{\text {th }}$ December 2018).

5. Huhmann MB, Cunningham RS. Importance of nutritional screening in treatment of cancer-related weight loss. Lancet Oncol 2005; 6: 334-343.

6. Tisdale MJ. Cachexia in cancer patients. Nat Rev Cancer 2002; 2: 862-871.

7. Fearon KC, Voss AC, Hustead DS. Definition of cancer cachexia: effect of weight loss, reduced food intake, and systemic inflammation on functional status and prognosis. Am J Clin Nutr 2006; 83: 1345-1350.

8. Caccialanza R, Cereda E, Pinto C, et al. Awareness and consideration of malnutrition among oncologists: Insights from an exploratory survey. Nutrition 2016; 32: 1028-1032.

9. Spiro A, Baldwin C, Patterson A, Thomas J, Andreyev HJN. The views and practice of oncologists towards nutritional support in patients receiving chemotherapy. Br J Cancer 2006; 95: 431-434.

10. Rauh S, Antonuzzo A, Bossi P, et al. Nutrition in patients with cancer: a new area for medical oncologists? A practising oncologist's interdisciplinary position paper. ESMO Open 2018; 3: e000345.

11. Caccialanza R, De Lorenzo F, Gianotti L, et al. The cancer patients' charter of rights for appropriate and prompt nutritional support. Nutritional Care Cancer 2017; 25: 3001-3004.

12. Institute of Medicine (IOM). Knowing what Works in Health Care: A Roadmap for the Nation. The National Academies Press, Washington DC 2008.

13. Mauri D, Tsiara A, Valachis A, Kalopita K, Tsali L, Tolis P, Polyzos NP. Cancer cachexia: global awareness and guideline implementation on the web. BMJ Support Palliat Care 2013; 3: 155-160.

14. Bonis PA, Pickens GT, Rind DM, et al. Association of a clinical knowledge support system with improved patient safety, reduced complications and shorter length of stay among Medicare beneficiaries in acute care hospitals in the United States. Int I Med Inform 2008; 77: 745-753.

15. Human Development reports. Human Development index (HDI) 2010 Rankings. http://hdr.undp.org/en/statistics/ (lastly accessed June 2011).

16. Association of Community Cancer Center (ACCC). Issues in Nutrition and Cancer: Update 2004. Oncology Issues 2004 Suppl; 19: s2-14. www.accc-cancer.org (lastly accessed June 2011).

17. National Comprehensive Cancer Network (NCCN). NCCN Clinical practice Guideline in Oncology. Palliative Care. PAL-11, and MS6. www.nccn.org (lastly accessed June 2011).

18. Oncology Nutrition Dietetic Practice Group. www.oncologynutrition.org (lastly accessed June 2011).

19. Canadian Association of Nurses in Oncology (CANO/ACIO). Anorexia guideline. www.cano-acio.org (lastly accessed June 2011).

20. European Palliative Care Research Collaborative (EPCRC). Clinical practice guidelines on cancer cachexia in advanced cancer patients with a focus on refractory cachexia. www.epcrc.org (lastly accessed June 2011).

21. European Association for Palliative Care (EAPC). Clinical practice guidelines on cancer cachexia in advanced cancer patients with a focus on refractory cachexia. www.eapcnet.org (lastly accessed June 2011).

22. Cancer Research UK (CRUK). Nutrition support in adults. Understanding NICE guidance - information for people who need nutrition support, their families and carers, and the public. NICE Clinical Guideline 32, February 2006. www.cancerresearchuk.org (lastly accessed June 2011).

23. National Institute of Health and Excellence. Nutrition support in adults. Understanding NICE guidance - information for people who need nutrition support, their families and carers, and the public. NICE clinical Guideline 32, February 2006. www.nice.org.uk (lastly accessed June 2011).

24. Swedish Cancer Society (SCS). Food for Cancer. www.cancerfonden.se (lastly accessed June 2011).

25. Italian Association of Cancer Patients. Neoplasia e perdita di peso che cosa fare? www.aimac.it (lastly accessed June 2011). 
26. Del Fabbro E, Inui A, Strasser F, Blum D, Suzuki H. Cancer Cachexia. Springer Healthcare Communications 2012

27. European Society for Medical Oncology (ESMO). https://oncologypro.esmo.org/content/download/98014/1726202/file/E-Learn ing-Pathophysiology-and-Management-of-Cancer-Cachexia. pdf\%20Esmo\%20pro\%202016 (lastly accessed June 2018).

28. European School of Oncology (ESO). https://academic.oup.com/ annonc/article/25/8/1492/271866 (lastly accessed March 2019).

29. European Cancer Patient Coalition (ECPC). http://www.ecpc.org/ images/updated\%20Draft\%20Consultation\%20Document.pdf (lastly accessed March 2019).

30. European Oncology Nursing Society (EONS). http://www.cancernurse.eu/documents/EONSnewsletter2006winter-en.pdf and http://www.cancernurse.eu/documents/EONS8PresentationBozzetti.pdf (lastly accessed March 2019).

31. European Palliative Care Research Collaborative (EPCRC). http:// www.epcrc.org is no further available, EPCRC guidelines can be accessed http://www.cancercachexia.com/literature-watch/43 clinical-practice-guidelines-on-cancer-cachexia-in-advanced-cancer (lastly accessed March 2019) and International Consensus with EPCRC participation is further available at https://www.sbno.com.br/ UploadsDoc/Apoio\%206.pdf (lastly accessed March 2019).

32. American Society of Clinical Oncology (ASCO). https://media4 asco.org/156/edbook/pdfs/EdBookAM201535e229.pdf (lastly accessed March 2019).

33. National Comprehensive Cancer Network (NCCN). https://www. nccn.org guideline: Palliative Care, subsection Cachexia/Anorexia (lastly accessed June 2018) now had been substitute by 2019 guideline (lastly accessed March 2019).

34. Cancer Care (CC). https://media.cancercare.org/publications/original/230-roundtable_lungcancer.pdf (lastly accessed March 2019), and https://www.cancercare.org/publications/140-coping with cancer-related_weight_changes_and_muscle_loss(lastlyaccessed March 2019).

35. National Cancer Institute (NCl) https://www.cancer.gov/aboutcancer/treatment/research/cachexia (lastly accessed March 2019) and https://www.cancer.gov/about-cancer/treatment/side-effects/ appetite-loss/nutrition-hp-pdq (lastly accessed March 2019).

36. Association of Community Cancer Centers (ACCC). www.accc-cancer.org/search-results?keywords=cachexia (lastly accessed March 2019).

37. Oncology Nutrition Dietetic Group (ADA). http://www.oncologynutrition.org (lastly accessed March 2019).

38. CPAC (Canadian Partnership Against Cancer) https://www.partnershipagainstcancer.ca/db-sage/sage20151277/ (lastly accessed March 2019) https://www.partnershipagainstcancer.ca/db-sage/ sage20131737/ (lastly accessed March 2019).

39. SEOM (Spanish Society of Medical Oncology) https://seom.org/ seomcms/images/stories/recursos/Art nutricion en cancer Seccion_CC_oct2017.pdf (lastly accessed March 2019).

40. Sociedad Española de Enfermería Oncológica (SEEO). https:// www.seeo.org/wp-content/uploads/2017/01/nutricion-II-completo.pdf, https://www.seeo.org/wp-content/uploads/2017/01/nutricion-III-completo.pdf, https://www.seeo.org/wp-content/uploads/ 2017/03/Nutricion-I-completo-final.pdf (lastly accessed March 2019).

41. Belgian Federation Against Cancer (FCC) https://www.cancer.be/ les-cancers/effets-secondaires/une-perte-de-poids-extr-me-narien-dagr-able (lastly accessed March 2019).

42. Cancer Research UK (CRUK). https://www.cancerresearchuk. org/about-cancer/coping/physically/diet-problems/types/ cachexia? ga=2.157697661.698474958.1547898455-1638504187. 1547898455, and https://www.cancerresearchuk.org/about-cancer/ coping/physically/diet-problems/managing/diet-in-advanced -cancer? ga=2.10848755.698474958.1547898455-1638504187. 1547898455, and https://www.cancerresearchuk.org/about-cancer/ coping/physically/diet-problems/research? ga= 2.181882208.698474958.1547898455-1638504187.1547898455 (lastly accessed March 2019).

43. National Institute of Health and Excellence (NICE) https:// www.nice.org.uk/guidance/ng85, and https://www.nice.org.uk/ researchrecommendation/cachexia-interventions-a-cohort-study- followed-by-phase-ii-and-iii-studies-should-be-undertaken-in-people-with-pancreatic-cancer-and-cachexia-or-pre-cachexia-to-compare-cachexia-assessment-methods-and-anti-cachexia-interventions-with-standard-care, and https://www.nice.org.uk/guidance/ indevelopment/gid-ta10105 (lastly accessed March 2019).

44. Italian Association of Cancer Patients (AIMAC). https://www. aimac.it/libretti-tumore/cancro-pancreas/gli-aspetti-nutrizionali-per-il-cancro-del-pancreas, and https://www.aimac.it/notizie-tumore/media/la-farmaconutrizione-un-aiuto-ai-pazienti-oncologici-per-una-migliore-qualita-di-vita (March 2019).

45. Italian Institute of Medical Oncology (AIOM). https://www.aiom. it/linee-guida/trattamento-e-prevenzione-della-cachessia-neoplastica-10/ (lastly accessed March 2019).

46. Chinese Anti-Cancer Association (CACA). http://www.caca.org.cn (lastly accessed March 2019).

47. Chinese Society of Clinical Oncology (CSCO). http://www.csco. ac.cn/ (lastly accessed March 2019).

48. Kaikani W, Bachmann P. Consequences of a comorbidity often neglected in oncology in oncology: malnutrition. Bull Cancer 2009; 96: 659-664.

49. Breivik H, Cherny N, Collett B, de Conno F, Filbet M, Foubert AJ, Cohen R, Dow L. Cancer-related pain: a pan-European survey of prevalence, treatment, and patient attitudes. Ann Oncol 2009; 20: 1420-1433.

50. Polyzos NP, Mauri D, Ioannidis JP. Guidelines on chemotherapy in advanced stage gynecological malignancies: an evaluation of 224 professional societies and organizations. PLoS One 2011; 6: e20106.

51. Polyzos NP, Valachis A, Mauri D, Ioannidis JPA. Industry involvement and baseline assumptions of cost-effectiveness analyses: diagnostic accuracy of the Papanicolaou test. CMAJ 2011; 183 : E337-343.

52. Mauri D, Kalopita K, Tsiara A, et al. Cancer Pain: global awareness and guideline implementation. Forum Clin Oncol 2013; 4: 19-31.

53. Human Development Reports. Human Development Index (HDI) 2017 Rankings. http://hdr.undp.org/en/statistics/ (lastly accessed June 2018)

\section{Address for correspondence}

Davide Maur

Department of Medical Oncology

University Hospital of loannina

Leof. Stavrou Niarchou

45500 loannina, Greece

e-mail: dvd.mauri@gmail.com

Submitted: 24.03 .2019

Accepted: 17.04.2019 


\section{Appendix 1. Identification of pertinent societies and caregivers}

\section{Internet searches:}

We performed internet searches (last search June 2011) involving possible combinations of nine subject matters ("cancer", "oncology", "medical oncology", "clinical oncology", "radiation oncology", "radiotherapy", "surgical oncology", "cancer research", "supportive oncology") with three terms for educational and policymaker societies ("society” or "association" or "organization") and 30 terms of geographic identifiers (10 pertaining to continents: "Asian”, "American”, "North American”, "South American”, "America Latina”, “African”, “European”, "Australian”, "Oceania”, "International"; 10 pertaining to eligible countries by the highest development index: 8 "Norway", "Australia", "New Zealand", "USA", "Ireland", "Liechtenstein", "Netherlands", "Canada", "Sweden", "Germany"; and 10 pertaining to countries with a long lasting tradition in oncology but not included in the top 10 highly developed countries: "Austria", "Belgium", "China”, “Denmark”, “France”, “Japan”, “Italy”, “UK”, "Spain”, “Switzerland”).

Due to notable economic and development differences between South and North American countries, the continental entities were separately searched and analyzed for North and South America. The first 100 results for each internet search were scrutinized. We included both societies with accessible Web pages and those whose presence was mentioned in some URL but did not have not a Web page or their link was not functional (under construction or not working). We scrutinized 144,000 Web pages during internet searches and identified 275 oncology societies and organizations covering a large array of oncology settings (educational/clinical/research/policymaker).

\section{Data extraction from eligible website}

From each pertinent oncology educational/professional/policymaker society and caregiver website we recorded its name, the URL, continent and/or country, subspecialty setting (medical oncology, surgical oncology, radiation oncology, cancer research) and whether they provided any cancer cachexia or malnutrition related guideline implementation. Whenever there was availability of an electronic search within the website, we used the terms "guidelines" or "recommendations" or "position statements". For each cachexia and/or malnutrition guideline retrieved we further addressed whether it was implemented for patients or for physicians and whether the grading of level of evidence of recommendations and the strength of recommendations were provided.

\section{Appendix 2. Cancer cachexia awareness} List of 270 scrutinized oncology societies/organizations

\section{- ACORN CRO}

- Africa Oxford Cancer Consortium

- African Cancer Organization

- African Organisation for Research and Training in Cancer

- African Radiation Oncology Group

- African Women's Cancer Awareness Association

- Alles Over Chemotherapie

- Alliance Mondiale Contre le cancer

- American Anti-Cancer Society

- American Association for Cancer Education

- American Association for Cancer Research

- American Brachytherapy Society

- American Cancer Society

- American College of Oncology Administrators

- American College of Radiation Oncology

- American Institute for Cancer Research

- American Society for Therapeutic Radiology and Oncology

- American Society of Clinical Oncology

- American Society of Preventive Oncology

- American-Italian Cancer Foundation

- Arbeitsgemeinschaft Internistische Onkologie

- Asian American Network for Cancer Awareness

- Asian Clinical Oncology Society

- Asian Federation of Organizations for Cancer Research and Control

- Asian Fund for Cancer Research

- Asian Oceania Clinical Oncological Society
- Asian Pacific Organization of Cancer Prevention

- Association for Directors of Radiation Oncology Programs

- Association for International Cancer Research

- Association of American Cancer Institutes

- Association of Cancer Executives

- Association of Community Cancer Centers

- Association of European Cancer Leagues

- Association of Freestanding Radiation Oncology Centers

- Association of Integrative Oncology and Chinese Medicine

- Association of Physician Assistants in Oncology

- Association of Residents in Radiation Oncology

- Australian Cancer Research Foundation

- Austrian Cancer Aid Society

- Austrian Cancer Association

- Austrian Society of Hematology and Oncology

- Austrian Society of Oncology

- Austrian Society of Oncology Pharmacy

- Austrian Society of Radiation Oncology

- Austrian Society of Surgical Oncology

- Belgian Association for Cancer Research

- Belgian Association for Radiotherapy and Oncology

- Belgian Federation Against Cancer

- Belgian Society of Medical Oncology

- Belgian Society of Surgical Oncology

- British Accelerator Science and Radiation Oncology Consortium

- British Association of Cancer Research 
- British Association of Cancer United Patients

- British Association of Surgical Oncology

- British Oncological Association

- British Oncology Pharmacy Association

- Canadian Association of General Practitioners in Oncology

- Canadian Association of Medical Oncologists

- Canadian Association of Nurses in Oncology

- Canadian Association of Pharmacy in Oncology

- Canadian Association of Provincial Cancer Agencies

- Canadian Association of Radiation Oncologists

- Canadian Cancer Action Network

- Canadian Cancer Advocacy Network

- Canadian Cancer Research Alliance

- Canadian Cancer Society / National Cancer Institute of Canada

- Canadian Oncology Societies

- Canadian Partnership Against Cancer

- Canadian Society for Surgical Oncology

- Cancer Advocacy Coalition of Canada

- Cancer Assistance Network

- Cancer Association of South Africa

- Cancer Australia

- Cancer Care

- Cancer Control New Zealand

- Cancer Council Australia

- Cancer Cure Foundation

- Cancer Federation

- Cancer Foundation of China / former Chinese Cancer Research Foundation

- Cancer Hope Network

- Cancer Patients Foundation

- Cancer Project

- Cancer Research Foundation of America

- Cancer Research Initiative of South Africa

- Cancer Research Institute

- Cancer Research Society of Canada

- Cancer Research UK

- Cancer society of New Zealand

- Cancer Support Association of Western Australia

- Cancer Support France

- Cancer Trials New Zealand

- Cancérologues Sans Frontières / Oncologists Without Borders

- Canteen Ireland

- Central European Cooperation Oncology Group

- China East Radiation Oncology Group

- Chinese Anti-Cancer Association

- Chinese Cancer Research Foundation

- Chinese Center for Disease Control and Prevention

- Chinese Medical Association

- Chinese Medical Association Society of Oncology

- Chinese Oncology Society (Taiwan).

- Chinese Preventive Medicine Association

- Chinese Society of Clinical Oncology

- Chinese Society of Therapeutic Radiology and Oncology / Chinese Society of Radiation Oncology

- Clinical Cancer Research Center (Switzerland)

- Clinical Oncology Society of Australia

- Commission on Cancer (CoC) Member organizations

- Community Oncology Alliance
- Concerted Action for Complementary and Alternative Medicine Assessment in the Cancer Field

- Conseils Pour la Chimiothérapie

- CRIS Foundation for Cancer Research

- Cure Cancer Australia Foundation

- Danish Cancer Society

- Danish Research School in Molecular Cancer Research

- Danish Society of Medical Oncology

- Dansk Selskab for Cancerforskning

- Dutch Association of Medical Oncology

- Dutch Association of Oncology Nurses (Vereniging Van Oncologie Verpleegkundigen)

- Dutch Belgian Hemato-Oncology Cooperative Group

- Dutch Cancer Society

- Dutch Society for Radiotherapy and Oncology

- Dutch Society of Oncology

- Dutch Society of Surgical Oncology

- Eastern Cooperative Oncology Group

- European Association for Cancer Education

- European Association for Cancer Research

- European Association for Palliative Care

- European CanCer Organisation

- European Cancer Prevention Organization

- European Masters Program in Radiation Sciences for Oncology

- European Organization for Research and Treatment of Cancer

- European Palliative Care Research Collaborative

- European School of Oncology

- European Society for Hyperthermic Oncology

- European Society for Medical Oncology

- European Society for Therapeutic Radiology and Oncology

- European Society of Cancer Immunology and Immunotherapy

- European Society of Oncology Pharmacy

- European Society of Surgical Oncology

- Fédération Nationale des Centres de Lutte Contre le Cancer

- Federation of Spanish Cancer Societies

- Fight Cancer Foundation

- Foundation Cancer Research Switzerland

- Foundation of Geriatric Oncology Netherlands

- Freesia Group for cancer charities Spain

- French Association for Therapeutic Research against Cancer

- French National Institute of Cancer

- French Society of Radiation Oncology

- French Society of surgical oncology

- German Cancer Aid

- German Cancer Research Center

- German Cancer Society

- German Society for Hematology and Oncology

- German Society of Radiation Oncology

- Intercultural Cancer Council

- Intercultural Cancer Council

- International Agency for Research on Cancer

- International Cancer Biomarker Consortium

- International Cancer Microenvironment Society

- International Cancer Rehabilitation Association

- International Network for Cancer Treatment and Research 
- International Organization for Cancer Prevention and Research

- International Society for Biological Therapy of Cancer

- International Society for Cell and Gene Therapy of Cancer

- International Society for Oncology and Biomarkers

- International Society of Cellular Oncology

- International Society of Intraoperative Radiation Therapy

- International Society of Oncology Pharmacy Practitioners

- International Union Against Cancer

- Ireland Cooperative Oncology Research Group

- Irish Association for Cancer Research

- Irish Association for Nurses in Oncology

- Irish Cancer Data Association

- Irish Cancer Society

- Irish Institute of Radiography and Radiation Therapy

- Irish Society of Medical Oncology

- Irish Society of Surgical Oncology

- Israel Cancer Association

- Italian Association for Cancer Research

- Italian Association for Radiation Oncology

- Italian Association of Cancer Patients

- Italian Cancer Society

- Italian Foundation for Cancer Research

- Italian Institute for Cancer Research and Treatment

- Italian Institute of Medical Oncology

- Italian League Against Cancer

- Italian Society for Surgical Oncology

- Japan Clinical Cancer Research Organization

- Japan Society of Clinical Oncology

- Japan Society of Therapeutic Radiology and Oncology

- Japanese Cancer Association

- Japanese Foundation for Cancer Research

- Japanese Organization of Radiotherapy Quality Management

- Japanese Society of Hyperthermic Oncology

- Japanese Society of Medical Oncology

- La Ligue Nationale Contre le Cancer

- L'Association pour la Recherche sur le Cancer (ARC)

- Latin American and Caribbean Society of Medical Oncology

- Latin American Association for Palliative Care

- Latin American Cancer Research Coalition

- Latin American Society for Therapeutic Radiation Oncology (ALATRO)

- Macmillan Cancer Support

- Medical Oncology Group of Australia

- Mediterranean School of Oncology

- Multinational Association of Supportive Care in Cancer

- National Association of Professional Cancer Coaches

- National Cancer Institute

- National Cancer Registrars Association

- National Cancer Research Institute

- National Cancer Research Network

- National Coalition for Cancer Survivorship

- National Comprehensive Cancer Network

- National Foundation for Cancer Research

- National Health and Medical Research Council

- National Institute of Health and Excellence

- New Zealand Society for Oncology

- Nordic Cancer Union
- Norwegian Cancer Society

- Norwegian Group on Inherited Cancer

- Oncology Nutrition Dietetic Group

- Organisation of European Cancer Institutes

- Organization for Oncology and Translational Research

- Prevent Cancer Foundation

- Radiation Therapy Oncology Group

- Royal Australian \& New Zealand College of Radiologists

- Scientific Association of Swiss Radiation Oncology

- Scottish Intercollegiate Guidelines Network

- Sino-American Network for Therapeutic Radiology and Oncology

- Sociedad Española de Enfermería Oncológica

- Societe Francaise du Cancer

- Society for Integrative Oncology

- Society of Radiation Oncology Administrations

- Society of Surgical Oncology

- South African Oncology Consortium

- South African Society of Clinical and Radiation Oncology

- South African Society of Medical Oncology

- South East Asian Radiation Oncology Group (SEAROG)

- Spanish Association Against Cancer

- Spanish Association for Cancer Research

- Spanish Association of Radiotherapy and Oncology

- Spanish Society of Chemotherapy

- Spanish Society of Medical Oncology:

- Spanish Society of Surgical Oncology

- Supportive and Rehabilitation Oncology

- Swedish Cancer Society

- Swedish Society of Oncology

- Swedish Surgical Society

- Swiss Bridge Foundation

- Swiss Cancer League, Swiss League Against Cancer

- Swiss Federation Against Cancer / Oncosuisse

- Swiss Group of Clinical Cancer Research

- Swiss Institute for Experimental Cancer Research

- Swiss Radiation Oncology Centers

- Swiss Society for Oncology

- Swiss Society of Medical Oncology

- Swiss Society of Surgery

- Taiwan Clinical Oncology Society

- The American College of Surgeons Oncology Group (ACOSOG)

- The Australian Organisation for Young People Living with Cancer

- The Austrian Cancer League

- The Cancer Information and Support Society

- The European Cancer Patient Coalition

- The European Oncology Nursing Society

- The European Society of Digestive Oncology

- The Japan Cancer Society

- The Japanese Association for Molecular Target Therapy of Cancer

- The New Zealand Association of Cancer Specialists

- The Royal College of Radiologists

- Trans Tasman Radiation Oncology Group

- World Cancer Research Fund International

- World Federation of Surgical Oncology Societies 